\title{
Uniform Magnetic Properties for an Ultrahigh-Density Lattice of Noninteracting Co Nanostructures
}

\author{
N. Weiss, ${ }^{1}$ T. Cren, ${ }^{1}$ M. Epple, ${ }^{1}$ S. Rusponi, ${ }^{1}$ G. Baudot, ${ }^{2}$ S. Rohart, ${ }^{2}$ A. Tejeda, ${ }^{2}$ V. Repain, ${ }^{2}$ S. Rousset, ${ }^{2}$ P. Ohresser, ${ }^{3}$ \\ F. Scheurer, ${ }^{4}$ P. Bencok, ${ }^{5}$ and H. Brune ${ }^{1}$ \\ ${ }^{1}$ Institut de Physique des Nanostructures, Ecole Polytechnique Fédérale de Lausanne (EPFL), CH-1015 Lausanne, Switzerland \\ ${ }^{2}$ Matériaux et Phénomenes Quantiques, Universités Paris 7, Laboratoire Associé au CNRS, UMR 7162, Case 7021, 2 Place Jussieu, \\ 75251 Paris Cedex 5, France \\ ${ }^{3}$ LURE, Université Paris Sud, UMR 130, batiment 209D, Orsay, France \\ ${ }^{4} I P C M S, U P R$ 7504, 23 rue du Loess, 67037 Strasbourg Cedex, France \\ ${ }^{5}$ ESRF, Boîte Postale 220, 38043 Grenoble Cedex, France
}

(Received 3 March 2005; published 6 October 2005)

\begin{abstract}
We report on the magnetic properties of two-dimensional Co nanoparticles arranged in macroscopically phase-coherent superlattices created by self-assembly on $\mathrm{Au}(788)$. Our particles have a density of 26 Tera/in ${ }^{2}\left(1\right.$ Tera $\left.=10^{12}\right)$, are monodomain, and have uniaxial out-of-plane anisotropy. The distribution of the magnetic anisotropy energies has a half width at half maximum of $17 \%$, a factor of 2 more narrow than the best results reported for superlattices of three-dimensional nanoparticles. Our data show the absence of magnetic interactions between the particles. $\mathrm{Co} / \mathrm{Au}(788)$ thus constitutes an ideal model system to explore the ultimate density limit of magnetic recording.
\end{abstract}

PACS numbers: 75.75.+a, 75.30.Gw, 81.16.Dn

The bit density on magnetic hard disks has been increasing at a constant pace for many years [1]. Besides the technological challenges, we face today the question of where downscaling ends from fundamental physics. This question can be addressed by studying periodic lattices of ferromagnetic monodomain particles, where each particle stores one magnetic bit. The high density requires out-ofplane magnetic anisotropy to minimize dipolar interactions among adjacent particles. Further, to optimize the signalto-noise ratio in read or write processes, the magnetic properties have to be uniform; i.e., the particles have to be uniaxial and the distributions of moments $M$, and magnetic anisotropy energies (MAEs), $K$, have to be narrow.

Chemical synthesis of self-assembled colloid particles has led to excellent size distributions with a half width at half maximum (HWHM) of $7 \%$ in diameter and $21 \%$ in volume, respectively $[1,2]$. However, these are accompanied by systematically much wider $K$ distributions $[3,4]$ partly caused by the random orientation of the particle's easy axes $[4,5]$ causing strong dipolar interactions. Colloid particles have obvious practical advantages, one of them being the high blocking temperatures [6]. However, for the outlined reasons, the ultimate density limit could not be explored with such systems so far. Alternatively, massselected magnetic 3D clusters [7] are monodisperse and may be soft-landed onto surfaces [8], but cannot be arranged into ordered arrays of equidistant magnetic units.

Self-assembly during atomic beam epitaxy on periodic strain relief patterns on single crystal surfaces has been shown to provide well ordered superlattices of 2D islands with narrow size distributions [9]. While such lattices are generally not in phase from terrace to terrace, a combination of epitaxial strain relief patterns and vicinal surfaces has been shown to lead to macroscopically phase-coherent lattices [10]. Former studies of the magnetic properties of metal islands on single crystal surfaces revealed that the spin-orbit interaction with the substrate may induce a strong enough magnetocrystalline anisotropy to yield a common out-of-plane easy magnetization axis for all islands [11-14]. Therefore we have today the tools to fabricate the required lattices of uniaxial monodomain particles.

In this Letter, we report on the first quantitative investigation of the magnetic properties of ultrahigh density lattices. We use kinetically controlled growth of Co on $\mathrm{Au}(788)$ to produce equidistant 2D Co monodomain particles with a density of 26 Tera dots $/ \mathrm{in}^{2}$. We present x-ray magnetic circular dichroism (XMCD) at the Co $L_{2,3}$ edge and magneto-optical Kerr effect (MOKE) measurements performed in situ with variable-temperature scanning tunneling microscopy (VT-STM) enabling a one-to-one correlation between sample morphology and magnetism as a function of coverage and temperature. The XMCD recorded as a function of sample orientation with respect to the applied magnetic field reveals uniaxial anisotropy and allows one to determine the Co spin and orbital moments. The temperature dependence of the zero-field susceptibility $\chi$ determined by means of MOKE shows an unprecedented narrow MAE distribution with HWHM of only $17 \%$. Finally, the $\chi$ data above the blocking temperature, and measurements on ensembles with bimodal distributions, demonstrate the absence of magnetic interactions between the particles.

The STM and zero-field susceptibility measurements were carried out in a UHV chamber in Lausanne with a geometry where molecular-beam epitaxy (MBE), VTSTM, and MOKE measurements are performed at a single sample position. The $\mathrm{Au}(788)$ crystal has been mechani- 
cally and electrochemically polished and was prepared in vacuum by repeated cycles of $\mathrm{Ar}$-ion sputtering $\left(1 \mathrm{keV}, 300 \mathrm{~K}, 1 \mu \mathrm{A} / \mathrm{cm}^{2}\right)$ and annealing to $900 \mathrm{~K}$. Co was deposited by $e$-beam evaporation from a high purity rod $(99.995 \%)$ at a background pressure below $8 \times 10^{-11} \mathrm{mbar}$ and with a flux of $0.30 \pm$ 0.03 monolayer $(\mathrm{ML}) / \mathrm{min}$. The XMCD measurements were performed in the total electron yield mode at beam line ID08 of the European Synchrotron Radiation Facility (ESRF) in Grenoble. The sample was prepared in situ under similar growth conditions as for the MOKE experiment, and the morphology of the superlattices was controlled with a VT-STM connected to the beam line UHV system.

The steps of the $\mathrm{Au}(788)$ surface are the energetically favorable $\{111\}$ microfacets. Because of elastic repulsions, they arrange equidistantly over the entire crystal [10]. The (111)-oriented terraces exhibit the $(\sqrt{3} \times 22)$ reconstruction [15], here with domain walls running perpendicular to the step edges and being aligned from terrace to terrace. Nucleation of Co takes place at the crossing of the domain walls and the steps $[10,16]$. As Fig. 1 shows, this leads to a well ordered superlattice of Co particles with uniform size $\left(\mathrm{HWHM}_{\mathrm{s}}=20 \%\right)$ which is phase coherent over the entire crystal.

The spin $\left(m_{\mathrm{S}}\right)$ and orbital $\left(m_{\mathrm{L}}\right)$ moments of the Co atoms in the 2D islands have been determined by means of XMCD for a superlattice with a coverage of $\theta=$ $0.35 \mathrm{ML}$, leading to a mean size of 120 atoms per island.

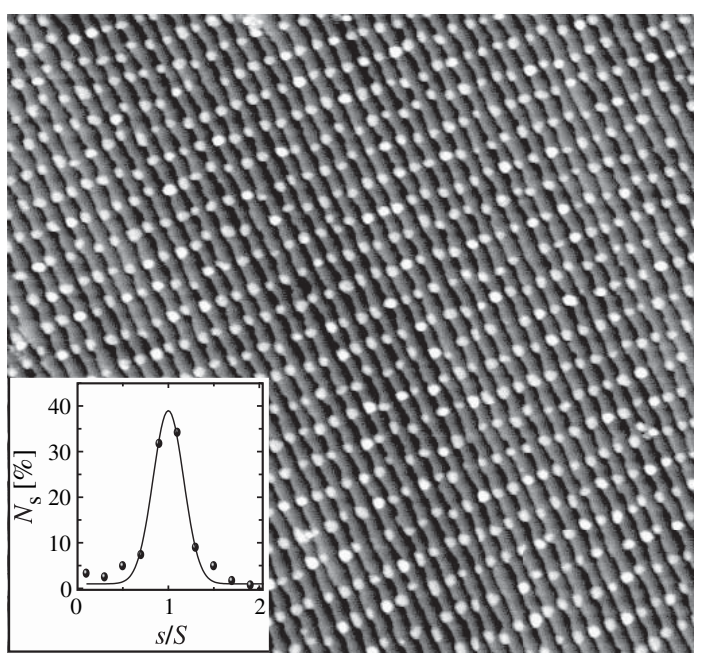

$30 \mathrm{~nm}$

FIG. 1. STM image showing 0.2 ML Co deposited on Au(788) at $130 \mathrm{~K}$ and annealed to $300 \mathrm{~K}$. Co forms bilayer islands organized into a long-range ordered superlattice with a unit cell of $3.5 \mathrm{~nm} \times 7.0 \mathrm{~nm}$, corresponding to a density of 26 Tera islands $/$ in $^{2}$. Inset: Size distribution with mean island size $S=70$ atoms and $\mathrm{HWHM}_{\mathrm{s}}=14$ atoms; $N_{\mathrm{s}}$ denotes the abundance of islands with size $s$.
Figure 2(a) shows the $\mathrm{x}$-ray absorption spectroscopy (XAS) data for both helicities recorded under normal incidence $\left[\gamma=0^{\circ}\right.$; see Fig. 2(b)] at the $\operatorname{Co} L_{2,3}$ absorption edges and at a magnetic field of $5 \mathrm{~T}$ saturating the sample. From the resulting XMCD spectrum, $m_{\mathrm{S}}$ and $m_{\mathrm{L}}$ can be derived by means of the sum rules [17,18]. From the angular dependence of $m_{\mathrm{L}}$, displayed in Fig. 2(c), it is seen that the sample's easy axis is within the error bars the surface normal, in agreement with the results for Co islands on $\mathrm{Au}(111)[12,19]$. However, the highest $m_{\mathrm{L}}$ value is found at $\gamma \simeq-15^{\circ}$, suggesting the easy axis to be slightly canted. This is, indeed, expected due to the fact that the Co islands are attached to substrate steps [20]. Since our data are only suggestive of a slight canting, and since this effect is small, we assume for simplicity in the following analysis an out-of-plane easy axis. The perpendicular and parallel component of the orbital moment, $m_{\mathrm{L}, \perp}$ and $m_{\mathrm{L}, \|}$, can then be calculated according to

$$
m_{\mathrm{L}}(\gamma)=m_{\mathrm{L}, \perp}+\left(m_{\mathrm{L}, \|}-m_{\mathrm{L}, \perp}\right) \sin ^{2}(\gamma) .
$$

We find $m_{\mathrm{L}, \perp}=(0.46 \pm 0.05) \mu_{\mathrm{B}}$ and $m_{\mathrm{L}, \|}-m_{\mathrm{L}, \perp}=$ $(0.11 \pm 0.01) \mu_{\mathrm{B}}$. Applying the sum rules and the formalism described in Refs. [17,18,21], we find a spin moment of $m_{\mathrm{S}}=(1.7 \pm 0.1) \mu_{\mathrm{B}}$ per Co atom close to the bulk value of $1.55 \mu_{\mathrm{B}}$ [22].

The transition from blocked to superparamagnetic behavior is characterized by the temperature dependence of the zero-field susceptibility $\chi(T)$. We determined this quantity as the field derivative of the polar Kerr intensity with the external field sweeping around zero $\left(\omega=2 s^{-1}\right)$. The amplitude of the field sweep is given by the compromise between signal-to-noise ratio and nonlinearities starting in the $M_{\text {tot }}(H)$ curves at high fields. When we label $M$ (a)

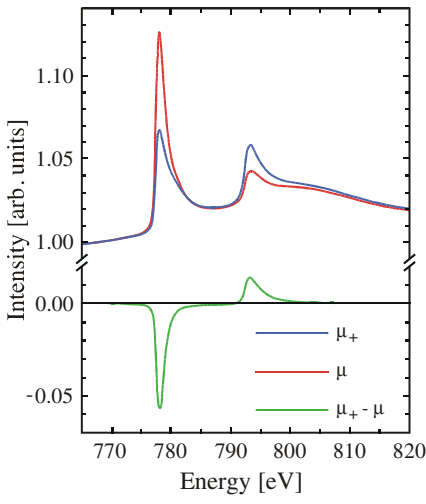

(b)

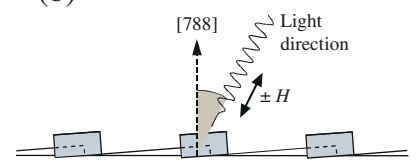

(c)

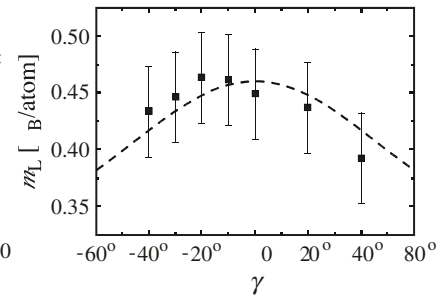

FIG. 2 (color online). (a) XAS spectra for $0.35 \mathrm{ML}$ Co on $\mathrm{Au}(788)$ taken at normal incidence $\left(\gamma=0^{\circ}\right)$, at $T=10 \mathrm{~K}$ and $B=5 \mathrm{~T} \quad\left(B_{\text {sat }}=2.5 \mathrm{~T}\right) . \mu_{+}$and $\mu_{-}$are left- and rightcircularly polarized incident light spectra, the difference being the XCMD signal. (b) Magnetic field and incident beam are parallel and form an angle $\gamma$ with the surface normal. (c) Orbital moment $m_{\mathrm{L}}$ as a function of $\gamma$. The line corresponds to the fit with Eq. (1). 
the magnetic moment of a particle, then linear response theory gives the upper field limit as $M H \ll k_{\mathrm{B}} T$ [23]. With typical values $\left(T=100 \mathrm{~K}, M=600 \mu_{\mathrm{B}}\right)$ this yields $H \ll$ $2500 \mathrm{Oe}$, justifying our amplitude of $100 \mathrm{Oe}$.

In order to address the MAE distribution of the Co islands, we consider a superlattice of slightly larger islands created by repeating the deposition and annealing sequence previously described in coverage steps of at most $0.3 \mathrm{ML}$. This procedure allows a linear increase of the island size preserving a narrow size distribution until the onset of coalescence $\left[\mathrm{HWHM}_{\mathrm{s}}=32 \%\right.$ for $\theta=0.75 \mathrm{ML}$; see Fig. 3(b)]. The zero-field susceptibility $\chi(T)$, shown in Fig. 3(c), has a sharp peak at $T_{\mathrm{b}} \sim 50 \mathrm{~K}$ marking the transition from the blocked state to the superparamagnetic one. This transition takes place in a narrow temperature window of about $15 \mathrm{~K}$, indicative of a very narrow MAE distribution.

For a quantitative description we note that each Co island is a monodomain particle [24]. The susceptibility of one particle at a given temperature depends on its magnetic moment, $M$, and on its MAE, $K$, in a way outlined in Ref. [23]. $M$ is the sum over the moments of the constituent Co atoms, $m_{\mathrm{Co}}=m_{\mathrm{L}}+m_{\mathrm{S}}$. Since $m_{\mathrm{S}}$ is largely independent of atomic coordination [25], and is much larger than $m_{\mathrm{L}}$, it is a good approximation to assume equal contributions from all the atoms in the cluster, $M=$ $s m_{\mathrm{Co}}$, with $s$ the cluster size and $m_{\mathrm{Co}}=2.2 \mu_{\mathrm{B}}$. For the attribution of $K$ to a given cluster, it is important to consider the coordination of the atoms [25]. Previous work on Co islands grown on $\mathrm{Au}(111)$ [12] and on $\mathrm{Pt}(111)$ [23] has (a)

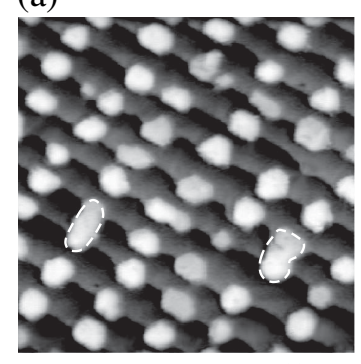

(c)

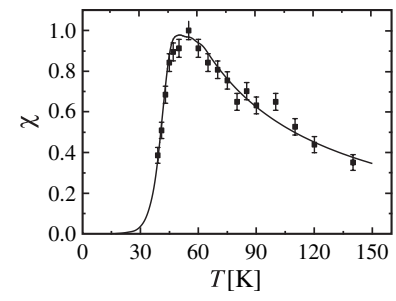

(b)

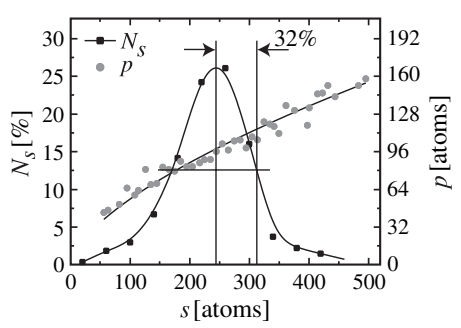

(d)

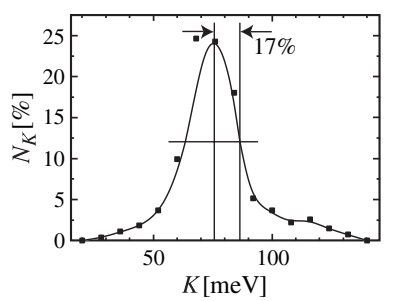

FIG. 3. (a) STM image showing the surface morphology for a Co coverage of $\theta=0.75 \mathrm{ML}$. Coalesced dots are encircled by dashed white lines. (b) Size and perimeter length distribution. (c) $\chi(T)$. The solid line represents the fit calculated by assuming $K$ to originate from the perimeter atoms. (d) MAE distribution used to fit $\chi(T)$ in (c). shown that $K$ is mainly determined by the perimeter atoms. We therefore assume $K=p k_{p}$ with $p$ being the number of perimeter atoms and $k_{p}$ their MAE. For simplicity, we also assumed that the perimeter atoms in the first and second atomic layers, as well as the small fraction of perimeter atoms in contact with the substrate steps, have equal $k_{p}$.

By means of careful analysis of the STM images, we derived the sizes and perimeter lengths for a statistical ensemble of more than 2000 islands. This information was used as input to calculate $\chi(T)$ of the particle ensemble by summing over the contribution of each particle [23]. Since the morphology is known, $k_{\mathrm{p}}$ is the only free parameter. It is seen from Fig. 3(c) that the calculated curve reproduces the experimental data very well, yielding $k_{\mathrm{p}}=$ $0.8 \pm 0.1 \mathrm{meV} /$ atom, in agreement with the value of $0.9 \pm$ $0.1 \mathrm{meV} /$ atom obtained for Co islands on $\mathrm{Pt}(111)$ [23] and with the estimate of $1.0 \pm 0.3 \mathrm{meV} /$ atom, which we derive from Ref. [12] for Co islands of comparable size on $\mathrm{Au}(111)$.

We can cross-check our MOKE and XMCD measurements by comparing the islands MAE measured with both techniques. The mean island size of the sample studied with XMCDis $s=120$ atoms, with $p=55$ atoms situated at the rim. Therefore $M=264 \mu_{\mathrm{B}}$ and from MOKE $K=$ $p k_{\mathrm{p}}=44 \pm 5 \mathrm{meV}$. Our XMCD results allow us to determine the anisotropy of $m_{\mathrm{L}}$, which is linked to the magnetocrystalline anisotropy energy per atom, $k_{\mathrm{MC}}$, by $[19,26-$ 28]

$$
k_{\mathrm{MC}}=-\alpha \frac{\xi}{4 \mu_{\mathrm{B}}}\left(m_{\mathrm{L}, \|}-m_{\mathrm{L}, \perp}\right)
$$

with $\xi \sim 70 \mathrm{meV}$ the Co spin-orbit coupling constant [19], and $\alpha$ accounting for the fact that the exchange splitting is generally smaller than the width of the band $[21,28]$. Since MOKE determines the total anisotropy $k=k_{\mathrm{MC}}+k_{\text {shape }}$, we subtract the shape anisotropy, which we estimate to be $k_{\text {shape }}=-0.08 \mathrm{meV} /$ atom, assuming circular island geometry. This yields a MOKE value of $k_{\mathrm{MC}}=0.45 \pm$ $0.04 \mathrm{meV} /$ atom. In order to meet this value with XMCD $\left(m_{\mathrm{L}, \|}-m_{\mathrm{L}, \perp}=(0.11 \pm 0.01) \mu_{\mathrm{B}}\right), \quad$ we $\quad$ find $\quad \alpha=$ $0.23 \pm 0.02$ confirming previously reported estimates of 0.2 [27].

Figure 3(d) shows the particles' MAE distribution derived from the perimeter length distribution and the value of $k_{p}$. Similar to the size distribution, the $K$ distribution also has a Gaussian shape; however, its $\mathrm{HWHM}_{\mathrm{K}}$ is with $17 \%$ almost a factor of 2 smaller than the one of the sizes of $32 \%$. This is explained by the MAE being largely given by the perimeter length, which in 2D has a distribution half as wide as the one of the size. The second remarkable feature is that the value of $17 \%$ is less than half of the best result so far achieved for colloid particles [3]. For the 3D colloid particles, the nonuniformity at the surface and the competition between several causes of anisotropy, such as faceting, strain, or shape anisotropy, may give rise to several 
(a)

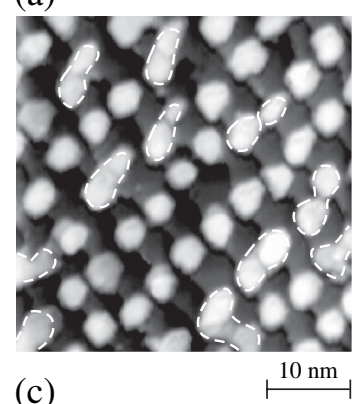

(c)

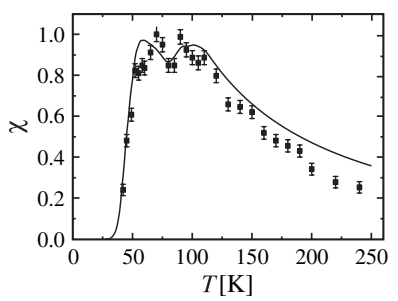

(b)

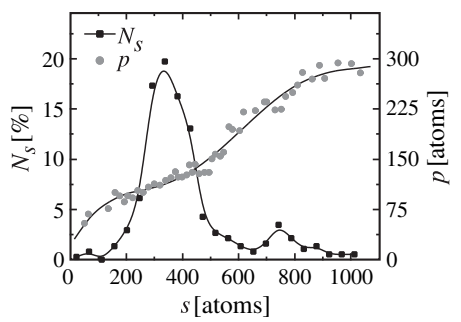

(d)

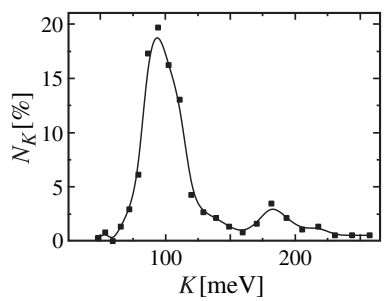

FIG. 4. (a) STM image showing the surface morphology for $\theta=1.1 \mathrm{ML}$. Coalesced particles are encircled by dashed white lines. (b) Size and perimeter length distributions. (c) $\chi(T)$. The solid line is the fit calculated by assuming $K$ to originate from the perimeter atoms. (d) MAE distribution used to fit $\chi(T)$ in (c).

easy axes per particle. In addition, these axes are randomly oriented from one particle to the next, and finally in some cases there are dipolar interactions, which altogether explains the relatively wide anisotropy energy distribution [4]. Our data demonstrate that ordered arrays of uniaxial out-of-plane nanostructures grown by MBE may achieve much more narrow MAE distributions than anticipated based on the size distribution alone.

Until now we tacitly assumed that dipolar interactions between the particles are absent. In the absence of interactions, $\chi(T)$ decays a little steeper than $1 / T$ for $T>T_{\mathrm{b}}$ [23], while interactions lead to a significantly flatter decrease [29]. Our data in Fig. 3(c) are in perfect agreement with the expected dependence of $\chi(T)$ for noninteracting particles. An independent observation demonstrating the absence of interactions is presented in Fig. 4 where we fabricated a sample with a bimodal size distribution due to the coexistence of noncoalesced and coalesced Co islands. For this sample $\chi(T)$ displays a clear double peak that is possible only if the macrospins of the two island sizes can fluctuate individually. The faster than $1 / T$ decrease of $\chi(T)$ above $180 \mathrm{~K}$ is probably due to the temperature dependence of the islands' magnetic moments, which decrease upon approaching the Curie temperature [30]. The fact that interactions are negligible can be rationalized by comparing the switching field $H_{\text {sw }}$ of a given island with the stray field $H_{\text {stray }}$ created by the presence of its neighbors. An upper bound to the stray field is obtained assuming all macrospins of the neighbors to be aligned. Considering

as an example a typical particle of Fig. 3(a) containing 260 atoms $\left(K \sim 65 \mathrm{meV}\right.$ and $\left.M=570 \mu_{\mathrm{B}}\right)$, we find $H_{\text {stray }} \sim 400 \mathrm{Oe}$. The temperature dependence of the switching field is given by $H_{\mathrm{sw}} \sim H_{0}\left(1-\sqrt{T / T_{\mathrm{b}}}\right)$ [31], where $H_{0}=2 \mathrm{~K} / \mathrm{M} \sim 4 \times 10^{4} \mathrm{Oe}$. This value is in agreement with $H_{0}=3.6 \times 10^{4}$ Oe derived from the hysteresis loops recorded at $20 \mathrm{~K}$ for 1500 atoms sized Co islands on $\mathrm{Au}(111)$ [32]. The switching field becomes comparable to the stray field only in the narrow interval $0.99 T_{\mathrm{b}}<T<T_{\mathrm{b}}$.

In conclusion, we have studied the magnetism of $\mathrm{Co}$ particles arranged into superlattices created by selfassembly on a $\mathrm{Au}(788)$ surface. Our particles are characterized by an unprecedented narrow MAE distribution and a common out-of-plane easy magnetization axis. Mutual magnetic interactions are negligible at a density of 26 Tera/in ${ }^{2}$.

[1] The Physics of Ultra-High-Density Magnetic Recording, Springer Series in Surface Science Vol. 41, edited by M. L. Plumer, J. van Ek, and D. Weller (Springer, Berlin, 2001).

[2] C. Petit, A. Taleb, and M. P. Pileni, J. Phys. Chem. B 103, 1805 (1999).

[3] S. I. Woods et al., Phys. Rev. Lett. 87, 137205 (2001).

[4] G. A. Held et al., Phys. Rev. B 64, 012408 (2001).

[5] C. T. Black et al., Science 290, 1131 (2000).

[6] S. Sun et al., Science 287, 1989 (2000).

[7] I. M. L. Billas, A. Châtelain, and W. A. D. Heer, Science 265, 1682 (1994).

[8] K. Bromann et al., Science 274, 956 (1996).

[9] H. Brune, Surf. Sci. Rep. 31, 121 (1998).

[10] V. Repain et al., Europhys. Lett. 58, 730 (2002).

[11] J. Shen et al., J. Appl. Phys. 81, 3901 (1997).

[12] H. A. Dürr et al., Phys. Rev. B 59, R701 (1999).

[13] S. Padovani et al., Phys. Rev. B 59, 11887 (1999).

[14] O. Fruchart et al., J. Magn. Magn. Mater. 239, 224 (2002).

[15] J. V. Barth et al., Phys. Rev. B 42, 9307 (1990).

[16] V. Repain et al., Mater. Sci. Eng. B 96, 178 (2002).

[17] B. T. Thole et al., Phys. Rev. Lett. 68, 1943 (1992).

[18] P. Carra et al., Phys. Rev. Lett. 70, 694 (1993).

[19] T. Koide et al., Phys. Rev. Lett. 87, 257201 (2001).

[20] P. Gambardella et al., Phys. Rev. Lett. 93, 077203 (2004).

[21] P. Ohresser et al., Phys. Rev. B 64, 104429 (2001).

[22] C. Chen et al., Phys. Rev. Lett. 75, 152 (1995).

[23] S. Rusponi et al., Nat. Mater. 2, 546 (2003).

[24] W. Wernsdorfer et al., Phys. Rev. Lett. 78, 1791 (1997).

[25] P. Gambardella et al., Science 300, 1130 (2003).

[26] P. Bruno, Phys. Rev. B 39, 865 (1989).

[27] D. Weller et al., Phys. Rev. Lett. 75, 3752 (1995).

[28] J. Stöhr, J. Magn. Magn. Mater. 200, 470 (1999).

[29] R. W. Chantrell et al., Phys. Rev. B 63, 024410 (2001).

[30] D. Zhang et al., Phys. Rev. B 58, 14167 (1998).

[31] M. P. Sharrock, IEEE Trans. Magn. 26, 193 (1990).

[32] E. Dudzik et al., J. Phys. Condens. Matter 11, 8445 (1999). 